\title{
Dietary energy sources and levels shift the multi-kingdom microbiota and functions in the rumen of lactating dairy cows
}

\author{
Tansol Park ${ }^{1 \dagger}$, Lu Ma ${ }^{2 \dagger}$, Ying $\mathrm{Ma}^{2}$, Xiaoqiao Zhou², Dengpan Bü ${ }^{2,3^{*}}$ and Zhongtang $\mathrm{Yu}^{1 *}$
}

\begin{abstract}
Background: Dietary energy source and level in lactation diets can profoundly affect milk yield and composition. Such dietary effects on lactation performance are underpinned by alteration of the rumen microbiota, of which bacteria, archaea, fungi, and protozoa may vary differently. However, few studies have examined all the four groups of rumen microbes. This study investigated the effect of both the level and source of dietary energy on rumen bacteria, archaea, fungi, and protozoa in the rumen of lactating dairy cows. A $2 \times 2$ factorial design resulted in four dietary treatments: low and high dietary energy levels (LE: 1.52-1.53; and HE: 1.71-1.72 Mcal/kg dry matter) and two dietary energy sources (GC: finely ground corn; and SFC: steam-flaked corn). We used a replicated $4 \times 4$ Latin square design using eight primiparous Chinese Holstein cows with each period lasting for $21 \mathrm{~d}$. The rumen microbiota was analyzed using metataxonomics based on kingdom-specific phylogenetic markers [16S rRNA gene for bacteria and archaea, $18 \mathrm{~S}$ rRNA gene for protozoa, and internally transcribed spacer 1 (ITS1) for fungi] followed with subsequent functional prediction using PICRUSt2.
\end{abstract}

Results: The GC resulted in a higher prokaryotic (bacterial and archaeal) species richness and Faith's phylogenetic diversity than SFC. For the eukaryotic (fungi and protozoa) microbiota, the LE diets led to significantly higher values of the above measurements than the HE diets. Among the major classified taxa, 23 genera across all the kingdoms differed in relative abundance between the two dietary energy levels, while only six genera (none being protozoal) were differentially abundant between the two energy sources. Based on prokaryotic amplicon sequence variants (ASVs) from all the samples, overall functional profiles predicted using PICRUSt2 differed significantly between LE and HE but not between the two energy sources. FishTaco analysis identified Ruminococcus and Coprococcus as the taxa potentially contributing to the enriched KEGG pathways for biosynthesis of amino acids and to the metabolisms of pyruvate, glycerophospholipid, and nicotinate and nicotinamide in the rumen of HE-fed cows. The co-occurrence networks were also affected by the dietary treatments, especially the LE and GC diets, resulting in distinct co-occurrence networks. Several microbial genera appeared to be strongly correlated with one or more lactation traits.

\footnotetext{
* Correspondence: budengpan@caas.cn; yu.226@osu.edu

${ }^{\dagger}$ Tansol Park and Lu Ma contributed equally to this work.

${ }^{2}$ The State Key Laboratory of Animal Nutrition, Institute of Animal Science, Chinese Academy of Agricultural Sciences, Beijing, P. R. China

'Department of Animal Sciences, The Ohio State University, Columbus, OH, USA

Full list of author information is available at the end of the article
}

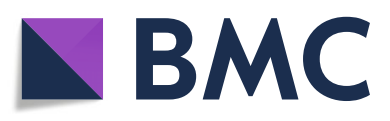

(c) The Author(s). 2020 Open Access This article is licensed under a Creative Commons Attribution 4.0 International License, which permits use, sharing, adaptation, distribution and reproduction in any medium or format, as long as you give appropriate credit to the original author(s) and the source, provide a link to the Creative Commons licence, and indicate if changes were made. The images or other third party material in this article are included in the article's Creative Commons licence, unless indicated otherwise in a credit line to the material. If material is not included in the article's Creative Commons licence and your intended use is not permitted by statutory regulation or exceeds the permitted use, you will need to obtain permission directly from the copyright holder. To view a copy of this licence, visit http://creativecommons.org/licenses/by/4.0/ The Creative Commons Public Domain Dedication waiver (http://creativecommons.org/publicdomain/zero/1.0/) applies to the data made available in this article, unless otherwise stated in a credit line to the data. 


\begin{abstract}
(Continued from previous page)
Conclusions: Dietary energy level affected the overall rumen multi-kingdom microbiota while little difference was noted between ground corn and steam-flaked corn. Some genera were also affected differently by the four dietary treatments, including genera that had been shown to be correlated with lactation performance or feed efficiency. The co-occurrence patterns among the genera exclusively found for each dietary treatment may suggest possible metabolic interactions specifically affected by the dietary treatment. Some of the major taxa were positively correlated to milk properties and may potentially serve as biomarkers of one or more lactation traits.
\end{abstract}

Keywords: Corn processing method, Dietary energy level, Functional profiles, Multi-kingdom, Rumen microbiota

\section{Introduction}

The rumen microbiota consists of bacteria, archaea, fungi, protozoa, and viruses, and its diversity and functional versatility enable robust utilization of various feed ingredients including recalcitrant plant wall materials, producing assimilable energy and nutrients required by ruminants [1]. Dietary interventions such as supplementation with feed additives and alterations of feed composition and digestibility are frequently used to optimize productivity (average daily gain and milk yield), improve product quality, and minimize animal health issues [2]. Indubitably, the effects and efficacy of these dietary interventions are made possible because of the rumen microbiota. Indeed, many of the production phenotypes of ruminant animals are associated with the rumen microbiota and its functionality [3-5]. However, few studies have analyzed bacteria, archaea, fungi, and protozoa together, missing the opportunity to examine how dietary interventions affect the interactions among these groups of prokaryotes and eukaryotes, which are important to the understanding of the microbial underpinning of treatment effects [6].

In dairy cows, the forage-to-concentrate ratio [7], source of forage [8], dietary energy source and level [9], and synchronization of dietary energy and nitrogen [10] are the major nutritional factors that determine milk yield and quality and are thus areas of extensive research. High-quality forage (e.g., corn silage and alfalfa hay) and energy-dense feed (e.g., corn) support high lactation performance, but in most countries outside of North America and Europe, these feed ingredients are limited in quantity and expensive, while low-quality forages, such as corn stover (CS) and rice straw, are abundant. Low-quality forages often decrease the metabolizable energy (ME) content of the diets because of their poor palatability, low digestibility, and low content of crude protein and non-fiber readily fermentable carbohydrates $[8,11,12]$. As a result, ruminal microbial protein (MP) synthesis and dietary energy supply to the host animals are inadequate to support high lactation performance $[8,12,13]$. Corn is the most energy-dense feed fed to dairy cows, and steam-flaking has been one of the most commonly used corn processing technologies to increase the digestibility of intact corn grains. Inclusion of steam-flaked corn (SFC) results in improved MP synthesis and lactation performance in lactating dairy cows $[14,15]$. Conceivably, the rumen microbiota will respond to the changes in source and level of forage and ME in the diet.

In a previous study investigating how dietary energy source and level affect feed digestibility, MP synthesis, and lactation performance of dairy cows, we found that total-tract apparent digestibility, milk yield, milk protein content and yield, and milk lactose yield all increased with increase in dietary energy, and when SFC replaced ground corn, milk yield, milk protein yield, and MP yield also increased [9]. We hypothesized that the observed differences in lactation performance might have resulted from the alteration of the rumen microbiota responding to the different energy sources and levels. The objective of this study was to examine how and to what extent the different sources and levels of dietary energy had affected the rumen microbiota and their interactions among themselves and with lactation performance phenotypes. To achieve a holistic view, bacteria, archaea, fungi, and protozoa were analyzed simultaneously using metataxonomics.

\section{Methods \\ Animals and diets}

The feeding experiment has been reported in a previous article [9]. Briefly, animal usage was approved by the Animal Care Advisory Committee of the Chinese Academy of Agricultural Sciences. A $2 \times 2$ factorial design resulted in four dietary treatments: low and high energy levels (LE: $1.52-1.53$; and HE: $1.71-1.72 \mathrm{Mcal} / \mathrm{kg}$ of dry matter) and two energy sources (GC: finely ground corn; and SFC: steam-flaked corn). The feed composition of the four dietary treatments was presented in Table S1. We used eight primiparous Chinese Holstein cows in a replicated $4 \times 4$ Latin square design with each period lasting for $21 \mathrm{~d}$. Four of the cows were each fitted with a rumen cannula and assigned to one of the four treatments. The animals were fed twice daily (06:30 and 16:30 h) for ad libitum intake, allowing for $5 \%$ orts, with free access to clean drinking water. Cows were milked thrice daily, 
at 07:00, 13:30, and 20:30 h. The rumen fluid samples were collected $3 \mathrm{~h}$ after the morning feeding on $\mathrm{d} 18$ using an oral stomach tube and then stored at $-80^{\circ} \mathrm{C}$ until metagenomic DNA extraction.

\section{DNA extraction and metataxonomic analysis of multi- kingdom rumen microbiota}

Metagenomic DNA from each rumen sample was extracted using the $\mathrm{RBB}+\mathrm{C}$ method [16]. The quality and quantity of the extracted metagenomic DNA were evaluated using a NanoDrop ND-2000 Spectrophotometer (Thermo Scientific, NanoDrop Technologies, Wilmington, DE, USA) followed by agarose gel $(1 \%$, wt/vol) electrophoresis. The rumen microbiota was analyzed using metataxonomics based on kingdom-specific phylogenetic markers (16S rRNA gene for bacteria and archaea, $18 \mathrm{~S}$ rRNA gene for protozoa, and internally transcribed spacer 1 (ITS1) for fungi). Briefly, one amplicon library for each rumen sample was prepared for each target microbial group by PCR amplification using the respective primer sets (Table S2). All the amplicon libraries had a unique barcode for multiplexing. The amplicon libraries for prokaryotes, protozoa, and fungi for each sample were pooled at 9: 0.5: 0.5 ratio first, resulting in one pooled amplicon library for each sample. Then, pooled amplicon libraries for all the samples were pooled again at equal ratio and sequenced using the $2 \times 300$ pairedend protocol on the Illumina MiSeq platform. Raw sequencing reads have been deposited in the NCBI Sequence Read Archive (SRA) under BioProject PRJNA523854.

The amplicon sequences were analyzed using QIIME2 with built-in commands and plugins (version 2019.4) [17]. Briefly, adapter and primer sequences were removed using Cutadapt [18] followed by quality filtering (Q-score $\geq 25$ ), denoising, merging, and chimeric sequence removal as done previously [19] using the DADA2 plugin [20]. Specifically for fungal sequences, ITSxpress [21] was used to delineate the ITS1 region followed by dereplication and chimera removal using q2-dada2's denoise-single method [20]. Amplicon sequence variants (ASVs) were clustered at $99 \%$ similarity. Taxonomic classifiers for each multi-kingdom were manually trained using the Naïve Bayes classifier [22] with the Greengenes $16 \mathrm{~S}$ reference database (13_8 version) for bacteria and archaea, the UNITE ITS reference sequences (10.10.2017 database) for fungi, and the Silva $18 \mathrm{~S}$ reference database (NR 132 version) for protozoa. Major phyla and genera each representing $\geq 0.1 \%$ of total sequences in at least one of the dietary treatments were discussed in this report.

Alpha-diversity measurements including richness (ASV, genera, and species), evenness, Faith's phylogenetic diversity, Shannon's diversity index, and Simpson's index were assessed based on the rarefied ASV BIOM tables. The overall microbiotas shaped by the different dietary treatments were compared using principal coordinates analysis (PCoA) based on the weighted UniFrac distances. With combined multikingdom compositional data at the genus level (major known genera only), Sparse Co-occurrence Network Investigation for Compositional data (SCNIC) was used to examine the correlation based on the co-occurrence and mutual-exclusion networks for each dietary treatment by computing Spearman correlation coefficients (https:// github.com/shafferm/SCNIC). Only significant interactions $(P<0.05)$ were further analyzed using Coexpression Differential Network Analysis (CoDiNA) [23] to identify specific microbial networks at each dietary treatment.

\section{Functional prediction and identification of taxonomic drivers}

Metabolic functions were predicted from 16S- and ITS1-based ASVs using Phylogenetic Investigation of Communities by Reconstruction of Unobserved States 2 (PICRUSt2) [24]. The identified functional features were determined using six different reference databases (Table 2). Overall functional dissimilarities among different dietary treatments were examined based on the relative abundance of predicted enzymes assigned to the Enzyme Nomenclature database (ENZYME, https://enzyme.expasy.org/) for both prokaryotic- and fungal-microbiota and then visualized using principal components analysis (PCA) based on BrayCurtis dissimilarities. Functional Shifts' Taxonomic Contributors (FishTaco) [25] was used to define both the differentially abundant KEGG pathways between two different dietary treatments and the corresponding taxonomic drivers of each detected pathway. Functional shifts of defined KEGG pathways attributable to major taxonomic drivers at the genus level of rumen prokaryotes were visualized using the FishTacoPlot $\mathrm{R}$ package (https:/github.com/borenstein-lab/ fishtaco-plot).

\section{Calculations and statistical analysis}

Alpha diversity measurements of each microbial community (bacterial and archaeal, fungal, or protozoal) and the counts of PICRUSt2-predicted functional features were statistically analyzed using the GLIMMIX procedure of SAS 9.4 (SAS Institute Inc., Cary, NC, USA):

$$
\mathrm{Y}_{\mathrm{ijk}}=\mathrm{u}+\mathrm{T}_{\mathrm{i}}+\mathrm{H}_{\mathrm{j}}+\mathrm{T}_{\mathrm{i}} \mathrm{H}_{\mathrm{j}}+\mathrm{e}_{\mathrm{ij}}
$$

where $\mu$ is the mean of alpha diversity measurements or the number of functional features, $T_{i}$ is the fixed effect 
of the dietary energy level $(i=1,2), H_{j}$ is the fixed effect of energy source $(j=1,2)$, and $e_{i j}$ is the residual error. $T_{i} H_{j}$ is the fixed effect of the interaction between ith dietary energy level and the $j^{\text {th }}$ energy source. Permutational multivariate analysis of variance (PERMANOVA) was used to further analyze the PCoA and PCA results to assess if each microbial community significantly differed between the two dietary energy levels or between the two energy sources using PAST3 [26] with 9999 random permutations. Differentially abundant microbial taxa between different dietary energy levels and between the two energy sources were identified using linear discriminant analysis (LDA) effect size (LEfSe) [27] with a logarithmic LDA score 2 as the cutoff. Pearson correlation coefficients between milk yield and milk components reported in the previous animal study [9] and the relative abundance of major known microbial genera determined in the present study were computed using the
PROC CORR procedure in SAS and visualized using the R (v.3.5.0) package "corrplot". Statistical significance was declared at $P \leq 0.05$ and tendency at $0.05<P \leq 0.10$.

\section{Results}

Dietary energy level affects alpha and beta diversity and co-occurrence interactions

After quality filtering and rarefaction, 36348, 1213, and 4981 sequences per sample were obtained for bacteria and archaea, fungi, and protozoa, respectively, which resulted in greater than $96.8 \%$ of Good's coverage for all the samples (data not shown). In the prokaryotic microbiota, HE significantly decreased the number of observed species and genera, while SFC corresponded to decreased numbers of observed ASVs and a lower Faith's phylogenetic diversity than GC (Table 1). The high dietary energy level significantly decreased the number of ASVs, species, genera, and Shannon diversity index while

Table 1 Alpha diversity measurements of the ruminal microbiota

\begin{tabular}{|c|c|c|c|c|c|c|c|c|c|}
\hline \multirow[t]{2}{*}{ Measurements } & \multicolumn{2}{|l|}{ LE } & \multicolumn{2}{|l|}{$\mathrm{HE}$} & \multirow[t]{2}{*}{ SEM } & \multicolumn{4}{|l|}{$P$-values } \\
\hline & GC & SFC & GC & SFC & & $\mathrm{NE}$ & ES & Period & $\mathrm{NE} \times \mathrm{ES}$ \\
\hline \multicolumn{10}{|l|}{ Bacteria and archaea } \\
\hline Observed ASVs & 1050 & 808 & 973 & 752 & 45.211 & 0.3456 & 0.0116 & 0.1646 & 0.7936 \\
\hline Observed genera & $110^{\mathrm{A}}$ & $100^{A B}$ & $100^{A B}$ & $91^{B}$ & 2.738 & 0.0368 & 0.0756 & 0.3117 & 0.8548 \\
\hline Observed species & $126^{\mathrm{A}}$ & $115^{\mathrm{AB}}$ & $113^{\mathrm{AB}}$ & $104^{\mathrm{B}}$ & 3.127 & 0.0360 & 0.1006 & 0.3115 & 0.7053 \\
\hline Shannon & 8.27 & 8.24 & 8.22 & 8.00 & 0.063 & 0.2532 & 0.3962 & 0.8501 & 0.5160 \\
\hline Simpson & 0.99 & 0.99 & 0.99 & 0.99 & 0.001 & 0.7580 & 0.5245 & $<.0001$ & 0.3247 \\
\hline Faith's PD & 57.66 & 50.46 & 54.08 & 48.65 & 1.429 & 0.2187 & 0.0285 & 0.1718 & 0.5956 \\
\hline Evenness & 0.83 & 0.86 & 0.83 & 0.84 & 0.006 & 0.4262 & 0.1314 & 0.5571 & 0.4853 \\
\hline \multicolumn{10}{|l|}{ Fungi } \\
\hline Observed ASVs & $97^{A B}$ & $101^{\mathrm{A}}$ & $71^{A B}$ & $68^{B}$ & 4.511 & 0.0008 & 0.9189 & 0.6974 & 0.7098 \\
\hline Observed genera & 50 & 52 & 41 & 40 & 1.947 & 0.0107 & 0.8837 & 0.7432 & 0.7498 \\
\hline Observed species & 63 & 64 & 47 & 49 & 2.931 & 0.0106 & 0.7737 & 0.8287 & 0.9804 \\
\hline Shannon & 5.33 & 5.46 & 4.91 & 5.03 & 0.075 & 0.0052 & 0.4194 & 0.3448 & 0.8850 \\
\hline Simpson & 0.95 & 0.96 & 0.94 & 0.95 & 0.003 & 0.0794 & 0.1340 & 0.1374 & 0.8218 \\
\hline Faith's PD & 25.75 & 26.58 & 20.36 & 22.03 & 0.989 & 0.0128 & 0.4742 & 0.6513 & 0.7740 \\
\hline Evenness & 0.81 & 0.82 & 0.81 & 0.83 & 0.007 & 0.7033 & 0.2685 & 0.0047 & 0.7557 \\
\hline \multicolumn{10}{|l|}{ Protozoa } \\
\hline Observed ASVs & $73^{\mathrm{A}}$ & $69^{A B}$ & $61^{A B}$ & $61^{B}$ & 2.275 & 0.0051 & 0.4157 & 0.6152 & 0.5865 \\
\hline Observed genera & 5 & 5 & 4 & 4 & 0.153 & 0.0131 & 0.6678 & 0.4967 & 0.3796 \\
\hline Observed species & $10^{\mathrm{A}}$ & $9^{A B}$ & $8^{B}$ & $9^{A B}$ & 0.243 & 0.0046 & 0.7940 & 0.5114 & 0.2616 \\
\hline Shannon & 4.79 & 4.62 & 4.61 & 4.54 & 0.116 & 0.4194 & 0.4437 & 0.6601 & 0.7803 \\
\hline Simpson & 0.93 & 0.91 & 0.94 & 0.93 & 0.012 & 0.4435 & 0.5476 & 0.4481 & 0.5636 \\
\hline Faith's PD & 0.87 & 0.82 & 0.72 & 0.76 & 0.024 & 0.0133 & 0.9700 & 0.7342 & 0.2230 \\
\hline Evenness & 0.77 & 0.76 & 0.78 & 0.77 & 0.015 & 0.7145 & 0.5960 & 0.5357 & 0.9009 \\
\hline
\end{tabular}

LE Low energy, HE High energy, GC Ground corn, SFC Steam-flaked corn, NE Effect of dietary energy level, ES Effect of energy sources, NE $\times E S$ Interaction between $\mathrm{NE}$ and ES

ASVs Amplicon sequence variants

Faith's PD Faith's phylogenetic diversity

Different superscripts in upper case within a row indicate significant difference $(P \leq 0.05)$. The $P$-values $\leq 0.05$ are bolded, while those $>0.05$ but $\leq 0.1$ are italicized 
increasing Faith's phylogenetic diversity of the fungal microbiota. As for the protozoal microbiota, HE decreased taxa richness (ASVs, species, and genera) and Faith's phylogenetic diversity. The two energy sources (GC vs. SFC) did not result in significant changes in any of the alpha diversity measurements of the fungal or the protozoal microbiotas. The PCoA based on the weighted UniFrac distance showed that the dietary energy level significantly affected the prokaryotic microbiota and tended to affect the fungal microbiota, but not the protozoa microbiota (Fig. 1). The two energy sources did not significantly alter the overall prokaryotic or eukaryotic microbiotas.

Compositional data-based network analysis identified 73 and 71 microbial nodes with 184 and 189 significant interactions among the identified genera $(P<0.05)$ for the two energy levels and the two energy sources, respectively (Fig. 2). When the significant co-occurrence and mutual exclusion interactions were compared between the two dietary energy levels or the two energy sources, more interactions were specifically assigned to LE or GC. Particularly, fungal populations were mainly responsible for those interactions.

\section{The two dietary energy levels, but not the two energy sources, affect some taxa of the rumen microbiota}

Irrespective of the dietary treatments, Bacteroidetes is the most predominant phylum accounting for at least $65 \%$ of the total prokaryotic microbiota, while Firmicutes and Proteobacteria came in second and third, respectively (Fig. S1). At the genus level, Prevotella represented about half of the entire prokaryotic community, and roughly $70 \%$ of the total prokaryotic sequences could be assigned to 24 major classified genera (each representing $\geq 0.1 \%$ of total sequences in at least one of the dietary treatments). Three major fungal phyla, Ascomycota, Basidiomycota, and Neocallimastigomycota, represented over $94 \%$ in all the dietary treatments. Two genera within Neocallimastigomycota (i.e., Orpinomyces and Piromyces) and Candida within Ascomycota were the most predominant fungal genera regardless of dietary treatments. Only five protozoal genera (i.e., Dasytricha, Entodinium, Isotricha, Ophryoscolex, and Polyplastron) were detected among the dairy cows. Overall, Entodinium was the most predominant protozoal genus, but its relative abundances varied from $52 \%$ to $73 \%$ depending on the dietary treatments.

The differentially abundant genera of the rumen microbiota between the different dietary energy levels and between the different dietary sources were identified using LEfSe (Fig. 3). At the phylum level of bacteria, the relative abundance of Bacteroidetes, Lentisphaerae, SR1, Verrucomicrobia, and Tenericutes were enriched in the cows fed the LE diet, whereas that of Proteobacteria and
Firmicutes were enriched in the cows fed the HE diets. The two dietary energy sources did not result in the enrichment of any of the major bacterial phyla. Three candidate genera within Bacteroidetes (i.e., CF231, YRC22, and BF311), one candidate genus within Firmicutes (i.e., RFN20), Anaeroplasma, and Sphaerochaeta were differentially abundant in the cows fed the LE diet. All the five differentially abundant bacterial genera identified in the cows fed the HE diets belonged to either Firmicutes or Proteobacteria. No fungal phyla were found to be differentially abundant, but eight major fungal genera were enriched in the cows fed the LE diet, while Saccharomyces was enriched in the cows fed the HE diets. Aspergillus and Hanseniaspora expanded relative abundance in the cows fed GC and SFC, respectively. The LE diet increased the relative abundance of Dasytricha and Polyplastron, while the HE diets increased the predominance of Entodinium. The two energy sources did not cause a significant difference in the relative abundance of any of the protozoal genera.

\section{The two dietary energy levels, but not the two energy sources, affect some predicted functions of rumen prokaryotes}

Various functional features were predicted from the $16 \mathrm{~S}$ rRNA gene sequences and ITS1 sequences using six different reference databases (Table 2). The dietary energy levels significantly affected the numbers of functional features, with an overall increase at least numerically by the LE diets irrespective of the energy sources. On the other hand, the two different energy sourced did not result in significant differences in any of the predicted functional features irrespective of the databases used. The overall distributions of prokaryotic and fungal enzymes predicted based on EC numbers were visualized in PCA plots (Fig. 4). Based on PERMANOVA analysis, the two dietary energy levels resulted in significantly different $(P=0.006)$ distributions of prokaryotic enzymes (based on EC numbers), while the two energy sources did not cause any difference $(P=0.678)$. The predicted categories of fungal enzymes did not differ between the two dietary energy levels or between the two energy sources. The relative abundances of the predicted enzymes were only different between the two dietary energy levels but not between the two dietary energy sources (Table 3). The two energy levels resulted in different abundance in 16 types of prokaryotic enzymes (based on EC numbers; including four EC 1, oxidoreductases; five EC 2, transferases; five EC 3, hydrolases; and two EC 5, isomerases) and 13 types of fungal enzymes (including one EC 1, oxidoreductases; three EC 2, transferases; six EC 3, hydrolases; two EC 2, lyases; and one EC 5, isomerases). Further analysis using 


\section{A. Bacteria \& methanogens}

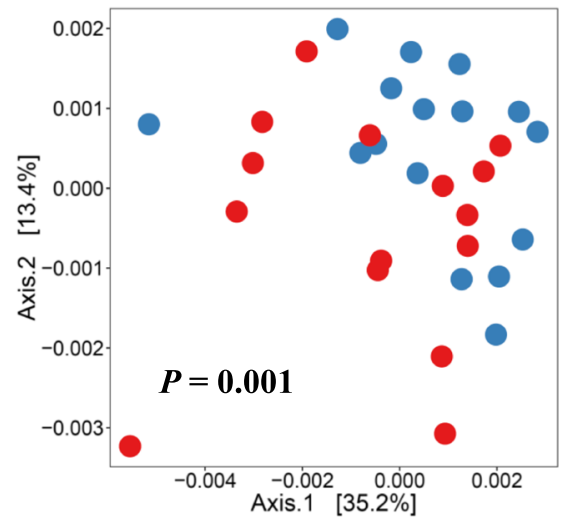

B. Fungi

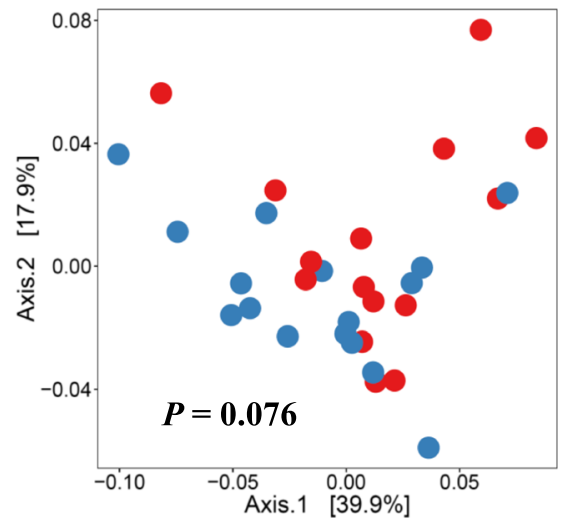

C. Protozoa

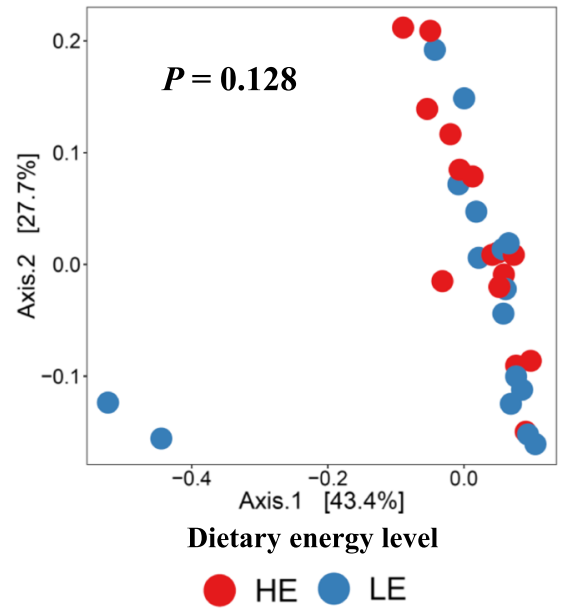

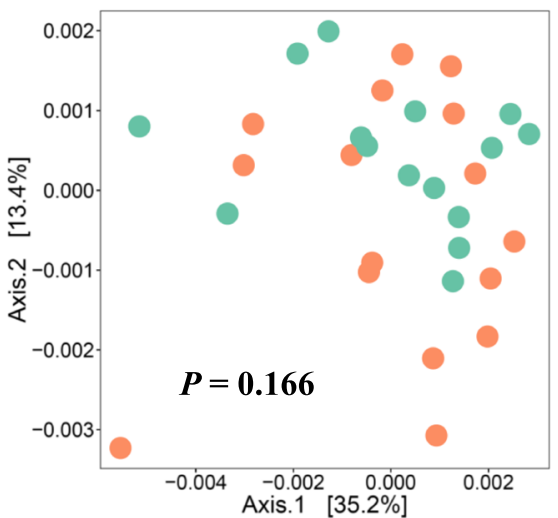
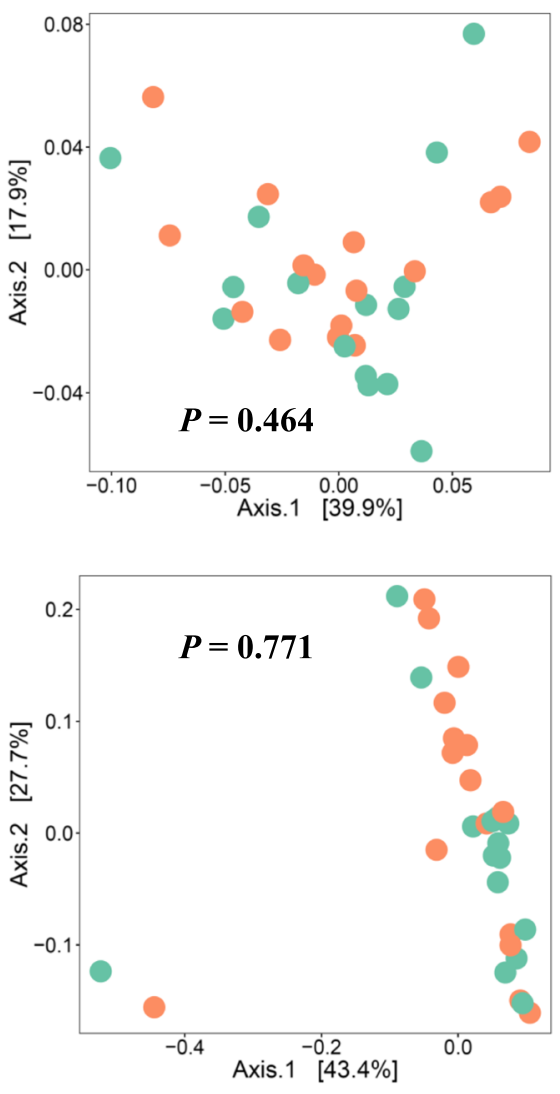

Dietary energy source

GC

SFC

Fig. 1 Plots of principal coordinates analysis (PCOA) of the rumen microbiota based on weighted UniFrac distance matrices. $H E$, high energy; LE, low energy; GC, ground corn; SFC, steam-flaked corn. P-values were based on PERMANOVA

FishTaco identified two KEGG pathways (i.e., ko00121, secondary bile acid biosynthesis; ko00120, primary bile acid biosynthesis) that were enriched by the LE diet, and the functional shifts were mainly associated with a candidate genus CF231 (Fig. 5a). In response to the HE diet, seven KEGG pathways (i.e., ko02010, ABC transporters; ko00290, valine, leucine and isoleucine biosynthesis; ko00300, lysine biosynthesis; ko00564, glycerophospholipid metabolism; ko00620, pyruvate metabolism; ko00400, phenylalanine, tyrosine and tryptophan biosynthesis; ko00760, nicotinate and nicotinamide metabolism) were enriched, and the enrichment was primarily contributed by Coprococcus and Ruminococcus (Fig. 5b). 


\section{LE vs. HE}

Overall: 73 nodes, 184 edges



LE-specific

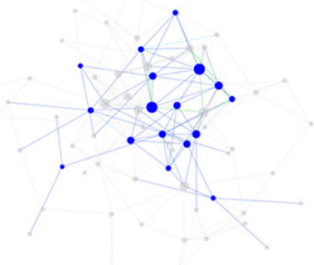

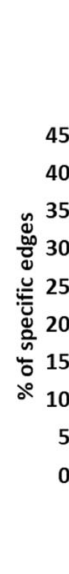

HE-specific



GC vs. SFC

Overall: 71 nodes, 189 edges

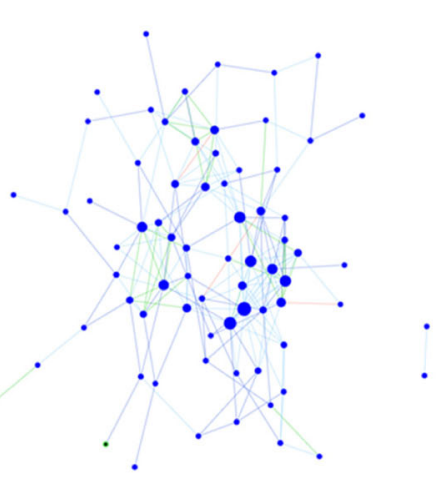

SFC-specific

Fig. 2 Co-occurrence network based on the significant correlations $(P<0.05)$ based on relative abundance of major genera (each having a relative abundance $\geq 0.1 \%$ in at least one of the dietary treatments) of rumen microbiota. The microbial taxa nodes exclusively found in each dietary treatment were also presented. The size of the nodes represents the sum of the weights of the interactions connected. HE, high energy; LE, low energy; GC, ground corn; SFC, steam-flaked corn. LE-specific nodes: one bacterial genus (Succinivibrio) and 16 fungal genera (Pyrenochaetopsis, Debaryomyces, Epicoccum, Orpinomyces, Gibberella, Bullera, Hannaella, Alternaria, Papiliotrema, Dioszegia, Penicillium, Cladosporium, Dipodascus, Saitozyma, Acremonium, and Sporobolomyces); HE-specific nodes: three bacterial genera (Prevotella, RFN20, and BF311), one fungal genus (Meyerozyma), and two protozoal genera (Dasytricha and Entodinium); GC-specific nodes: 10 fungal genera (Erythrobasidium, Sarocladium, Papiliotrema, Vishniacozyma, Acremonium, Sporobolomyces, Phaeosphaeria, Gibberella, Saitozyma, and Cladosporium) and one protozoal genus (Entodinium); SFC-specific nodes: one bacterial genus (Ruminobacter) and four fungal genera (Hanseniaspora, Cyberlindnera, Kurtzmanomyces, and Alternaria)

\section{Several taxa of bacteria, fungi, and protozoa exhibit correlation to lactation traits}

Significant and strong correlations $(|r|>0.5, P<0.05)$ between some of the lactation traits and several major genera were found (Fig. 6). Coprococcus, Shuttleworthia, and Ruminococcus, all of which were increased by the HE diets, were positively correlated with one or more of the lactation traits, either proportion or yield of major milk components, whereas Sphaerochaeta, which was increased in the LE diets, exhibited a negative correlation with both milk protein content and yield. Mitsuokella, Ruminobacter, Hanseniaspora (a fungal genus), which were enriched by or specifically associated with the SFC diets, also showed a positive correlation with several lactation traits. The fungal genus Saitozyma showed a negative correlation with milk lactose yield, while the protozoal genus Isotricha has a positive correlation with milk yield.

\section{Discussion}

Many nutritional factors affect the quality and quantity of milk produced by dairy cows [28]. Dietary manipulation is most commonly used to maximize lactation performance [2], which includes forage-toconcentrate ratio, grain processing, feed particle size, and forage length (e.g., [29]). Milk production by dairy cows is a highly energy-dependent process [30]. Indeed, in our previous study, the HE diets significantly increased milk protein content and the yield of milk, milk protein, and lactose although they did not affect milk fat or lactose [9]. The yield of milk and milk protein was also increased by SFC in the cows fed the LE diets. It was shown that the HE diets increased crude MP yield and total-tract apparent digestibility, and crude MP yield was also increased by SFC in the cows fed the LE diets. Apparently, lactation performance is strongly associated with rumen microbiota, yet, few studies have 

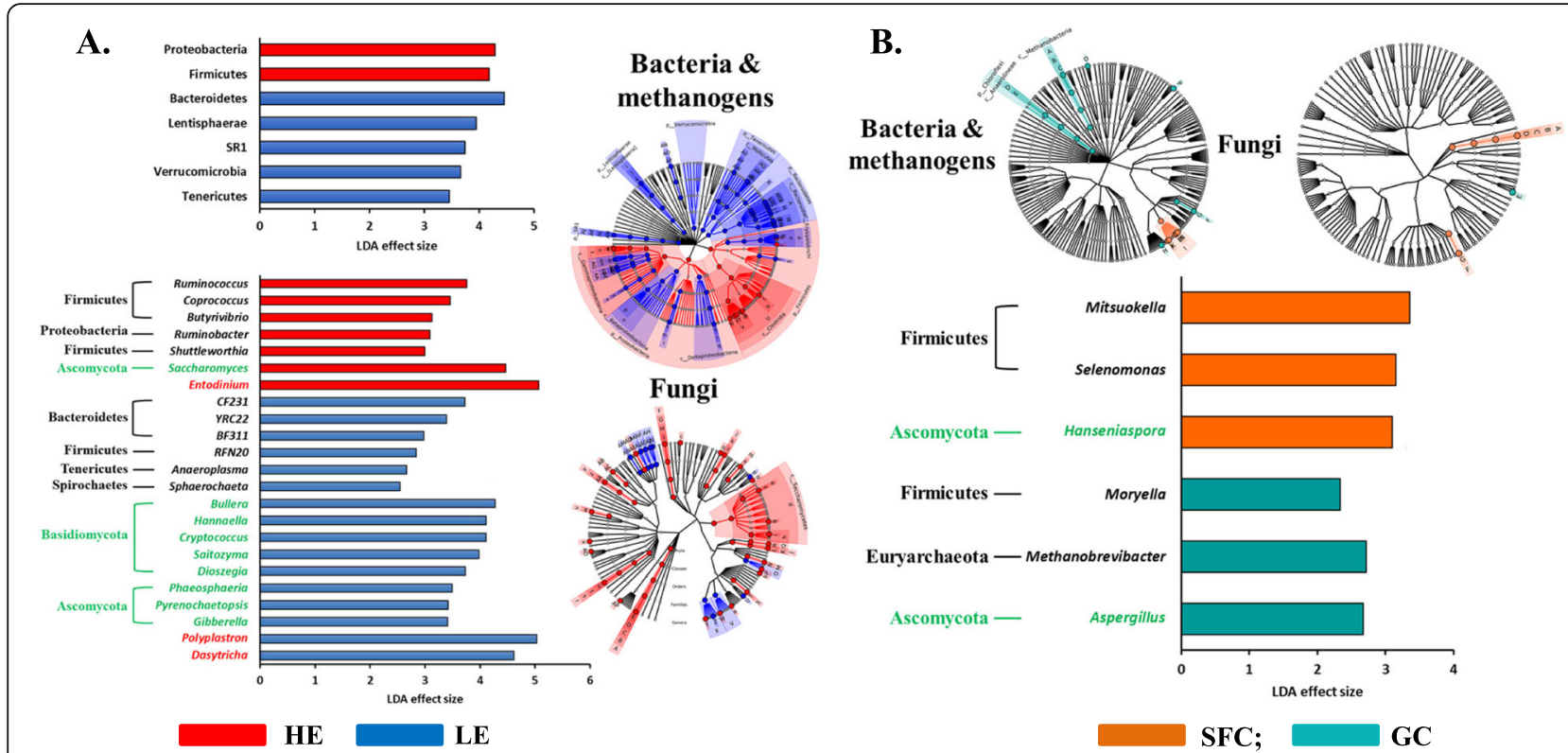

Fig. 3 Differentially abundant microbial genera as affected by energy levels (a) and energy sources (b), which were detected using LEfSe with an LDA effect size $\geq 2$. Only major classified taxa (each representing $\geq 0.1 \%$ in at least one of the dietary treatments) were visualized with additional taxonomic lineages of prokaryotes and fungi embedded in a respective cladogram. HE, high energy; LE, low energy; GC, ground corn; SFC, steam-flaked corn

focused on the effect of dietary energy level or source on rumen microbial shifts even though many studies have investigated the effect of the forage-toconcentrate ratio. Focusing on how both dietary energy source and level affect the multi-kingdom microbiota in dairy cows that showed different lactation performance [9], we identified taxa of rumen microbes that might be associated with some of the important lactation traits as affected by dietary energy level (1.53 vs. $1.72 \mathrm{Mcal} / \mathrm{kg}$ of dry matter) but not energy source (ground vs. steam-flaked corns).
Effect of dietary energy source and level on the prokaryotic, fungal, and protozoal rumen microbiota

The two different dietary energy levels resulted in differences in the diversity of the rumen prokaryotic microbiota, with the LE diets increasing species and genus richness. The positive correlation between species richness and the fiber content in diets has been demonstrated in several studies on dairy heifers and cows [31-33]. The increase in species richness by the LE diets is consistent with the above studies and corroborates the needs for concerted

Table 2 Predicted KEGG hierarchies (orthologs, modules, and pathways), Protein families (Pfam), Clusters of Orthologous Genes (COG), Enzyme Classification numbers (EC numbers), and MetaCyc pathways counts in each treatment

\begin{tabular}{|c|c|c|c|c|c|c|c|c|c|}
\hline \multirow[t]{2}{*}{ Measurements } & \multicolumn{2}{|l|}{$\underline{L E}$} & \multicolumn{2}{|l|}{$\mathrm{HE}$} & \multirow[t]{2}{*}{ SEM } & \multicolumn{4}{|l|}{$P$-values } \\
\hline & $\overline{G C}$ & SFC & $\overline{G C}$ & SFC & & $\overline{\mathrm{NE}}$ & ES & Period & $\mathrm{NE} \times \mathrm{ES}$ \\
\hline KEGG orthologs & 5731 & 5563 & 5351 & 5290 & 67 & 0.0126 & 0.3778 & 0.5038 & 0.6221 \\
\hline KEGG modules & 282 & 278 & 280 & 276 & 1 & 0.5080 & 0.1011 & 0.5542 & 0.9846 \\
\hline KEGG pathways & $145^{\mathrm{A}}$ & $143^{\mathrm{AB}}$ & $140^{B}$ & $141^{A B}$ & 1 & 0.0059 & 0.8504 & 0.3523 & 0.2589 \\
\hline PFAM & $6757^{a}$ & $6595^{\mathrm{ab}}$ & $6384^{\mathrm{ab}}$ & $6329^{b}$ & 68 & 0.0120 & 0.3980 & 0.3932 & 0.6092 \\
\hline $\operatorname{COG}$ & $4181^{a}$ & $4111^{\mathrm{ab}}$ & $4064^{\mathrm{ab}}$ & $4045^{b}$ & 22 & 0.0283 & 0.2666 & 0.7715 & 0.5084 \\
\hline EC numbers & $1816^{\mathrm{A}}$ & $1757^{\mathrm{AB}}$ & $1715^{\mathrm{AB}}$ & $1697^{\mathrm{B}}$ & 16 & 0.0096 & 0.2191 & 0.3870 & 0.4541 \\
\hline Fungal EC numbers & $989^{A}$ & $984^{\mathrm{AB}}$ & $972^{A B}$ & $966^{\mathrm{B}}$ & 3 & 0.0016 & 0.3540 & 0.3209 & 0.9354 \\
\hline MetaCyc pathways & $369^{A}$ & $361^{A B}$ & $343^{B}$ & $345^{A B}$ & 4 & 0.0035 & 0.6464 & 0.5974 & 0.4276 \\
\hline
\end{tabular}

LE Low energy, HE High energy, GC ground corn, SFC Steam-flaked corn, NE Effect of dietary energy level, ES effect of energy sources, NE $\times E S$ Interaction between NE and ES

Different superscripts within a row indicate significant difference $(P \leq 0.05)$, while different superscripts in lower case indicate tendency $(P \leq 0.1)$. The $P$-values $\leq 0.05$ are bolded 


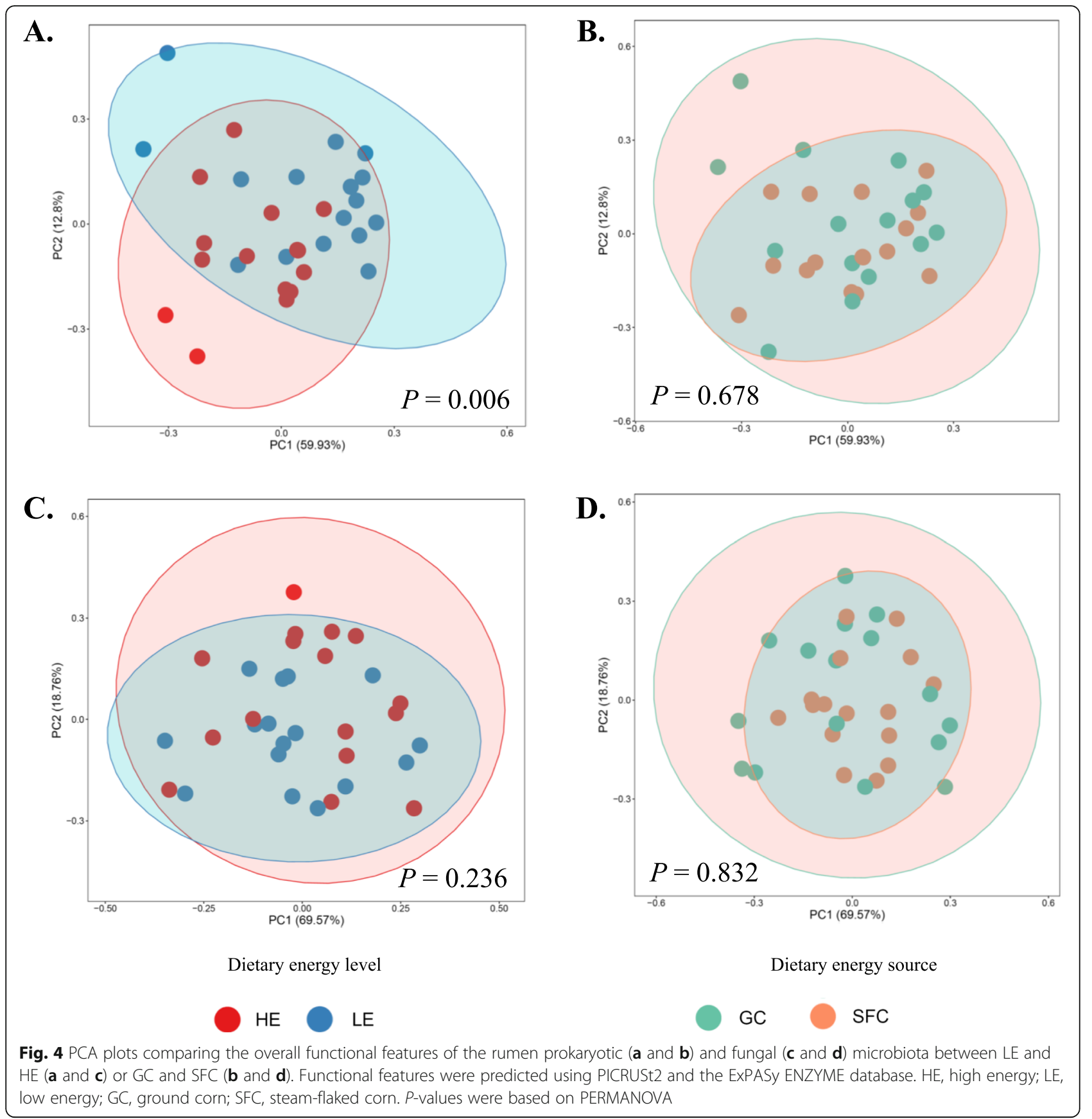

actions of a diverse microbiota to effectively digest recalcitrant feed fiber. The two energy sources only led to a significant difference in ASV richness and phylogenetic diversity, with the GC increasing both alpha diversity measurements. Although ASVs may not necessarily represent strains of microbes, the similar effects of both the energy source and energy level on species and genus richness, but not on ASV richness, may suggest that the two energy sources might have caused the difference in richness at the subspecies level. Based on our literature search, no study has been reported that examined the effect of ground vs. steam-flaked corns on rumen microbiota. It remains to be determined if the differences in digestibility between ground corn and steam-flaked corn may select different amylolytic bacteria. The two energy levels also shaped the fungal microbiota differently, though to a lesser extent than prokaryotic microbiota. The difference in fiber content between the two energy levels is likely attributable to the observed fungal effect. The relatively small effect of energy level on the fungal microbiota and the 
Table 3 Differentially abundant KEGG pathways affected by energy levels which were detected using LEfSe with an LDA effect size $\geq 2.0$

\begin{tabular}{|c|c|c|c|c|c|c|c|c|}
\hline \multirow[t]{2}{*}{ Item } & \multirow[t]{2}{*}{ EC numbers } & \multicolumn{2}{|c|}{ Relative abundance, $\%$} & \multirow[t]{2}{*}{ SEM } & \multirow[t]{2}{*}{ Class } & \multirow[t]{2}{*}{ LDA score } & \multirow[t]{2}{*}{$P$-value } & \multirow[t]{2}{*}{ Description } \\
\hline & & LE & HE & & & & & \\
\hline \multirow[t]{16}{*}{ Prokaryotic } & EC 1.2.7.11 & 0.506 & 0.467 & 0.007 & LE & 2.312 & 0.009 & 2-oxoacid oxidoreductase (ferredoxin) \\
\hline & EC 1.2.7.3 & 0.762 & 0.704 & 0.011 & LE & 2.483 & 0.013 & 2-oxoglutarate synthase \\
\hline & EC 1.3.5.1 & 0.271 & 0.248 & 0.004 & LE & 2.075 & 0.001 & Succinate dehydrogenase (quinone) \\
\hline & EC 1.6.5.3 & 1.333 & 1.279 & 0.010 & LE & 2.424 & 0.006 & NADH:ubiquinone reductase $\left(\mathrm{H}^{+}\right.$-translocating) \\
\hline & EC 2.7.7.13 & 0.291 & 0.270 & 0.004 & LE & 2.025 & 0.011 & Mannose-1-phosphate guanylyltransferase \\
\hline & EC 3.2.1.23 & 0.585 & 0.549 & 0.008 & LE & 2.249 & 0.020 & Beta-galactosidase \\
\hline & EC 3.2.1.51 & 0.382 & 0.352 & 0.006 & LE & 2.175 & 0.013 & Alpha-L-fucosidase \\
\hline & EC 3.4.21.102 & 0.400 & 0.377 & 0.004 & LE & 2.062 & 0.003 & C-terminal processing peptidase \\
\hline & EC 5.2.1.8 & 1.219 & 1.185 & 0.008 & LE & 2.195 & 0.018 & Peptidylprolyl isomerase \\
\hline & EC 5.4.99.2 & 0.181 & 0.156 & 0.004 & LE & 2.104 & 0.001 & Methylmalonyl-CoA mutase \\
\hline & EC 2.2.1.6 & 0.298 & 0.324 & 0.004 & HE & 2.130 & 0 & Acetolactate synthase \\
\hline & EC 2.3.1.15 & 0.094 & 0.115 & 0.004 & HE & 2.037 & 0.004 & Glycerol-3-phosphate 1-O-acyltransferase \\
\hline & EC 2.7.1.69 & 0.141 & 0.181 & 0.011 & HE & 2.303 & 0.009 & Protein-N(pi)-phosphohistidine-sugar phosphotransferase \\
\hline & EC 2.7.13.3 & 0.477 & 0.513 & 0.009 & HE & 2.247 & 0.009 & Histidine kinase \\
\hline & EC 3.4.16.4 & 0.261 & 0.286 & 0.003 & HE & 2.075 & 0 & Serine-type D-Ala-D-Ala carboxypeptidase \\
\hline & EC 3.6.3.17 & 0.108 & 0.128 & 0.006 & HE & 2.002 & 0.011 & Monosaccharide-transporting ATPase \\
\hline \multirow[t]{13}{*}{ Fungal } & EC 2.3.1.48 & 1.149 & 1.060 & 0.015 & LE & 2.646 & 0.006 & Histone acetyltransferase \\
\hline & EC 2.4.1.16 & 0.536 & 0.486 & 0.013 & LE & 2.396 & 0.019 & Chitin synthase \\
\hline & EC 3.2.1.18 & 1.825 & 1.736 & 0.021 & LE & 2.659 & 0.010 & Exo-alpha-sialidase \\
\hline & EC 3.2.1.3 & 2.166 & 1.922 & 0.052 & LE & 3.088 & 0.024 & Glucan 1,4-alpha-glucosidase \\
\hline & EC 4.6.1.2 & 0.063 & 0.042 & 0.007 & LE & 2.025 & 0.032 & Guanylate cyclase \\
\hline & EC 5.2.1.8 & 1.883 & 1.756 & 0.030 & LE & 2.803 & 0.012 & Peptidylprolyl isomerase \\
\hline & EC 1.14.13.12 & 0.077 & 0.123 & 0.011 & HE & 2.381 & 0.042 & Benzoate 4-monooxygenase \\
\hline & EC 2.3.1.85 & 0.036 & 0.060 & 0.006 & HE & 2.104 & 0.035 & Fatty-acid synthase \\
\hline & EC 3.1.1.3 & 0.509 & 0.530 & 0.005 & HE & 2.035 & 0.029 & Triacylglycerol lipase \\
\hline & EC 3.2.1.58 & 0.486 & 0.539 & 0.012 & $\mathrm{HE}$ & 2.419 & 0.003 & Glucan 1,3-beta-glucosidase \\
\hline & EC 3.4.17.21 & 0.149 & 0.172 & 0.005 & HE & 2.043 & 0.013 & Glutamate carboxypeptidase II \\
\hline & EC 3.5.1.4 & 0.271 & 0.305 & 0.010 & HE & 2.244 & 0.046 & Amidase \\
\hline & EC 4.1.1.1 & 0.129 & 0.155 & 0.004 & HE & 2.104 & 0.001 & Pyruvate decarboxylase \\
\hline
\end{tabular}

LE Low energy, HE High energy

No functional features were differed by energy sources

lack of impact on the protozoal microbiota are consistent with a previous report that the overall rumen protozoal and fungal microbiotas were slightly more stable than the prokaryotic microbiota in dairy cows fed diets with different forage-to-concentrate ratios [33]. The two energy sources did not cause much difference in the rumen microbiota, prokaryotic, fungal, or protozoal. This may be attributed to the lack of significant difference in digestibility between ground and the steam-flaked corns [9]. Collectively, these results suggest that under the dietary treatments of the present study, the dietary levels had a greater impact on the rumen microbiota than energy source (ground vs. steam-flaked corns).

\section{Effects of energy source and level on individual taxa of} the rumen microbiota

Ruminobacter and Shuttleworthia were increased by the HE diets, which can be explained by their ability to utilize starch [34, 35]. Ruminococcus, Coprococcus, Butyrivibrio, and Shuttleworthia, all of which are within the phylum Firmicutes, were considered as the core genera of rumen bacteria in large dairy cohorts [36]. Their relative abundance was increased by the HE diets. 




B.

ABC transporters .

Valine, leucine and isoleucine biosynthesis

Lysine biosynthesis Glycerophospholipid
metabolism

Pyruvate metabolism -

Phenylalanine, tyrosine and tryptophan biosynthesis

Nicotinate and nicotinamide metabolism ${ }^{-}$

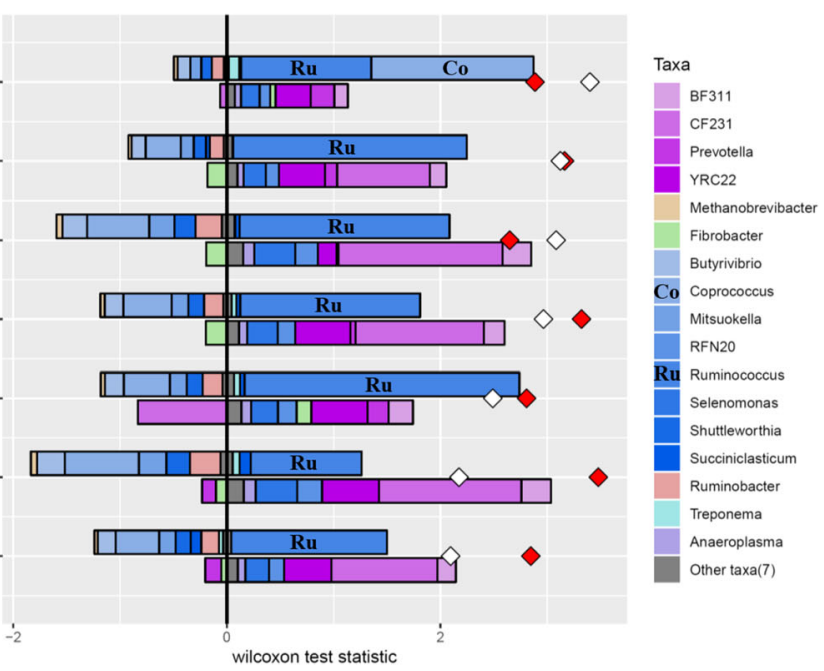

Fig. 5 Taxonomic drivers of differentially abundant KEGG pathways detected with FishTaco (only those with a z-score $\geq 2$ of Wilcoxon rank-sum test are shown). Only the major prokaryotic genera (each having a relative abundance $\geq 0.1 \%$ in at least one of the dietary treatments) were included in the PICRUSt2 and FishTaco analyses. a taxonomic drivers and KEGG pathways exclusively found in the cows fed LE diets; $\mathbf{b}$ taxonomic drivers and KEGG pathways exclusively found in the cows fed LE diets. The two energy sources did not result in significant difference in the predicted KEGG pathways

Ruminococcus and Butyrivibrio are generally considered as fibrolytic bacteria in the rumen, but some of their species also are amylolytic [37, 38], such as R. bromii and B. fibrisolvens, both of which could increase their growth in response to starch-rich diets [39]. However, those two bacterial genera were more frequently prevalent in cows fed forage-based diets [40]. Therefore, absolute quantification of several representative species of those major culturable bacteria is required to determine their population shifts under different dietary treatments. Three uncultured/unidentified candidate genera within the phylum Bacteroidetes, including CF231, YRC22, and BF311, were increased by the LE diets. Those three genera were significantly more predominant in the rumen of hay-fed goats compared to high-grain fed goats [41] and high-producing dairy cows (except for BF311) [42]. Another candidate genus (i.e., RFN20) within Firmicutes was also increased by the LE diets. These candidate genera were repeatedly found as major taxa, but the lack of cultured representatives and information on their metabolism hinders the interpretation of their shifts under given dietary treatments. The increased relative abundance of Sphaerochaeta and Anaeroplasma, both of which can use starch but not fiber, was consistent with the report on sheep fed corn stover [43] and on Holstein heifers fed a diet with increasing forage-to-concentrate ratio [33]. Moryella, which utilizes glucose, galactose, maltose, and ribose, but not starch 


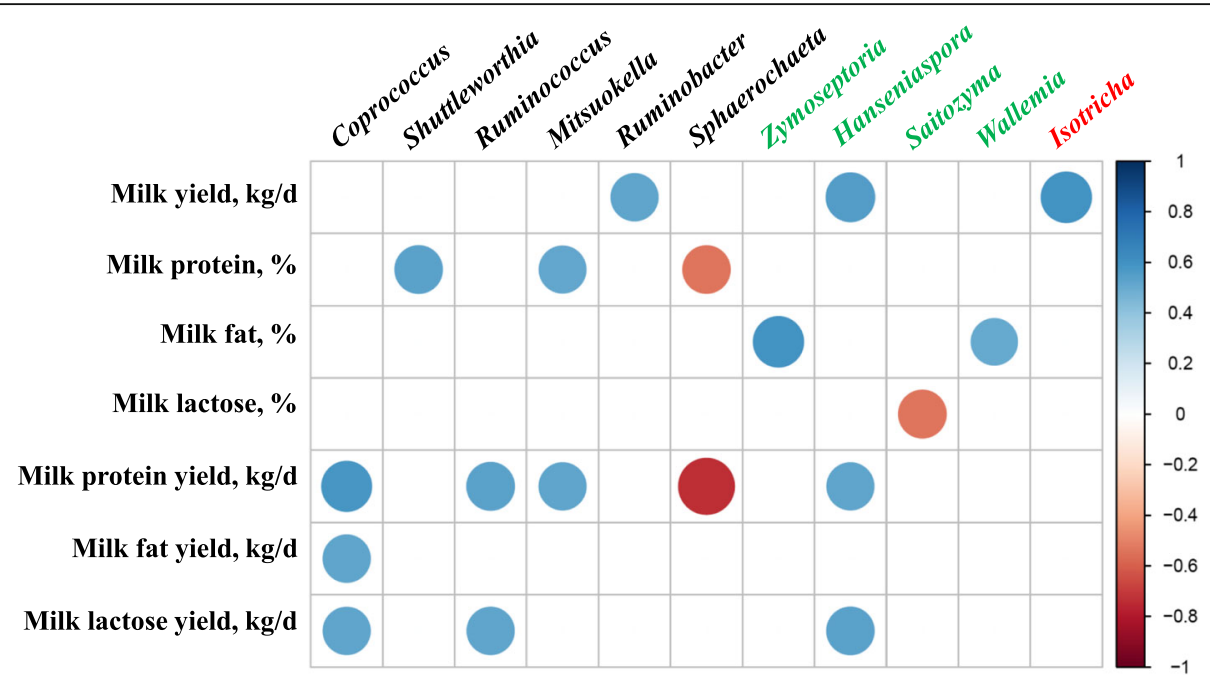

Fig. 6 Correlations between major classified genera (each having a relative abundance $\geq 0.1 \%$ in at least one of the dietary treatments; prokaryotes, fungi, and protozoa are labeled in black, green, and red, respectively) and milk quality and lactation performances. Only those with significant and strong correlation coefficients $(|r|>0.5, P<0.05)$ were visualized. The degree of each correlation was shown both with the size and intensity of the color based on the color key on the right side

[44], was previously linked to milk fatty acid profiles [45] and milk fat content [36]. In our previous study, however, milk fatty acid profiles were not determined and the milk fat was not affected by either energy source or level [9]. Other factors besides readily fermentable energy sources or levels might probably also shift its population. The increased relative abundances of two genera within Firmicutes, Mitsuokella, and Selenomonas, observed in the cows fed the SFC diets might be partially explained by increased digestibility of the steam-flaked corn compared to the ground corn. The readily fermentable SFC might have provided better niches for the starch-degrading bacteria resulting in increased lactation traits including the yield of fat-corrected milk (FCM), energy-corrected milk (ECM), milk protein, lactose, and content of milk protein and non-fat solid compared to the ground corn [9].

Saccharomyces was the only fungal genus having a higher relative abundance in the cows fed the HE diets compared to the LE diets. The increase of yeast is consistent with its occurrence in silage and the rumen of grain-fed cows [46]. The LE diets increased the predominance of eight genera of fungi, but interestingly, none of them is members of the phylum Neocallimastigomycota, to which all the six recognized and candidate genera of rumen fungi belong [47]. Based on our literature search, only Cryptococcus has been detected in the rumen of goats [48] and musk oxen [49]. Few studies have targetedly analyzed the fungi in ruminant nutritional studies. Future research can further determine their occurrence and potential function in the rumen. The two energy sources only affected two genera of fungi, with
Hanseniaspora being enriched by the SFC diets and Aspergillus being enriched by the GC diets. As a genus of yeast related to Saccharomyces, Hanseniaspora might have been increased due to the increased digestibility of SFC. The ability to use different carbohydrates including cellulose [50] might explain the higher predominance of Aspergillus resulted from the GC diets compared to the SFC diets. The higher relative abundance of Methanobrevibacter in the rumen of the GC-fed cows could enhance the fungal activity to break down the plant materials, as suggested in another study [51]. However, the differences of fungal profiles among the dietary treatments were possibly caused by the transient contamination from the forage intake which has been reported in a previous study [52]. Thus, the metabolic contribution of some of the fungi observed in the rumen might be limited and need to be interpreted with caution.

The differential relative abundance of Polyplastron and Entodinium in response to the LE and the HE diets, respectively, was possibly due to the different levels of fiber contents in the diets as previously reported in Holstein heifers [33]. Polyplastron is one of the largest entodiniomorphs which can engulf and digest fibrous feed particles inside its cells using its cellulases [53]. The dominance of the amylolytic Entodinium spp. has been repeatedly observed in dairy cattle fed high-grain diets $[33,54]$. However, Dasytricha is considered a saccharolytic protozoa genus with no known cellulolytic ability [55]. Besides the present study, only one study reported an increase in Dasytricha predominance by high forage diets [56]. Using refaunated dairy goats as a model, one 
study showed a negative correlation between Dasytricha and MP synthesis [57]. The higher predominance of this protozoal genus and the lower lactation performance of the cows fed the LE diets [9] corroborate the aforementioned study [57]. Future research is warranted to further investigate how diets affect Dasytricha and its relationship with MP synthesis in the rumen.

\section{Functional features shaped by different dietary energy levels and sources}

As expected from the differential prokaryotic microbiota diversity and composition, dietary energy levels significantly affected the overall predicted functional features. Besides, a greater number of functional features were predicted in the rumen microbiota of the cows fed the LE diets compared to the HE diets, irrespective of the reference databases used. On the other hand, the two energy sources (ground vs. steam-flaked corns) did not cause any significant difference in the predicted prokaryotic functions. This may be attributable to the lack of sensitivity or resolution (the data were based on relative abundance) the analysis methods or the high functional redundancy in the rumen microbiota [58]. However, the LE diets significantly increased the number of functional features compared to the HE diets. Furthermore, LEfSe analysis showed differentially abundant enzymes only between the two different dietary energy levels, and some of them are enzymes involved in carbohydrate metabolisms (e.g., 2-oxoacid oxidoreductase, 2-oxoglutarate synthase, succinate dehydrogenase, protein-N(pi)-phosphohistidine-sugar phosphotransferase, monosaccharidetransporting ATPase, glucan 1,4-alpha-glucosidase, pyruvate decarboxylase, and glucan 1,3-beta-glucosidase). The increased recalcitrance of the LE diets has probably created more niches and the requirement for more complex metabolic functions to digest the increased forage components. The recently updated fungal reference genome database in PICRUSt2 allows the prediction of fungal functions from ITS1 sequence data [24]. Unfortunately, the functional prediction from rumen protozoa is still not possible because of the lack of genomic information for any of the rumen protozoa.

\section{Microbial taxa potentially driving the difference in the predicted functional pathways between the two dietary energy levels}

To gain insights into the microbial underpinning of nutritional of functional changes, the microbes to which the observed changes can be attributable need to be identified. However, it is still difficult with complex high-dimensional microbiota data. FishTaco is an analytical and computational framework that integrates comparative taxonomic and functional analysis results to accurately quantify taxa contributing to disease- associated functional shifts [25]. It has been used in many studies on human microbiota, but only in one study on the rumen microbiota [59]. In the present study, CF231, a candidate genus-level taxon of the family Paraprevotellaceae, was identified to be the potential taxon contributing to the synthesis of primary and secondary bile acids in the rumen of the cows fed the LE diets. The prevalence of CF231 in the rumen was reported previously [36], and its abundance was negatively correlated to milk yield [42] but positively correlated with milk protein content [36]. Enriched secondary bile acid biosynthesis was found in beef cattle with high residual feed intake in a metatranscriptomic study of rumen microbiota [60]. Given the limited amount of bile acids present in the rumen, the inferred relationship between bile acid metabolism in the rumen and feed efficiency or milk production of dairy cows is intriguing. The unknown metabolism of CF231 adds more mist to this mystery.

Enriched $\mathrm{ABC}$ transporters and pyruvate metabolism in response to the HE diets possibly related to the increased sugar transport and VFA production, respectively [61]. The abundance of those pathways was also shown to vary depending on the dietary treatment [62], methane yield [63], and feed efficiency [64]. Furthermore, biosynthesis pathways for seven amino acids and glycerophospholipid metabolism were also enriched by the HE diets. The increased gene abundance of amino acid biosynthesis can be explained as an increase in the growth of the taxonomic drivers by the HE diets and is consistent with the increase of lactation performance in the cows fed the HE diet [9]. Ruminococcus was the contributor that explains several HE-enriched pathways: biosynthesis of amino acids (valine, leucine, isoleucine, lysine, phenylalanine, and tyrosine, tryptophan) and metabolism of glycerophospholipid, pyruvate, nicotinate, and nicotinamide. Both Ruminococcus and Coprococcus contributed to the enrichment of $\mathrm{ABC}$ transporters. When we searched the IMG database [65] that contains 29 sequenced genome of Ruminococcus isolates of rumen origin and did the KEGG annotation using the KEGG Automatic Annotation Server [66], we found all the HE-enriched KEGG pathways (data not shown). Coprococcus, which uses the acrylate pathway for propionate production from lactate, was more predominant in efficient dairy cows [5]. Also, Lachnospiraceae, which harbors several genera of ruminal bacteria including Coprococcus, Butyrivibrio, and Shuttleworthia, was previously shown to be positively correlated with milk production and milk protein content, though not with milk fat content [67]. It has been consistently recognized that the Firmicutes-to-Bacteroidetes ratio was positively linked to feed efficiency, milk production, and milk fat yield [4]. The increase of Lachnospiraceae abundance 
corresponded to a significantly higher $(P=0.0005)$ Firmicutes-to-Bacteroidetes ratio (data not shown) in the HE diet group, and this might be partially associated with the efficient conversion of the HE diets to milk observed in the previous study [9].

\section{Effect of energy source and level on microbial interactions}

The two dietary energy sources and levels resulted in some differences in the co-occurrence networks, with considerably more fungal nodes being found for the $\mathrm{LE}$ and the GC diets. Each of the four diets also resulted in a specific co-occurrence network. Although the co-occurrence networks were based on correlation in relative abundance, some of them may reflect biological interactions. However, it is not feasible to verify or test if a co-occurrence relationship is the consequence of biological interactions. Among the major fungal microbiota, the cellulolytic genera Acremonium, Cladosporium, and Penicillium [68, 69] were exclusively found in the co-occurrence networks corresponding to the LE or the $\mathrm{GC}$ diets, but those fungi are not commonly found in the rumen. Their actual residence and contribution need further verification. Among the known common ruminal fungi, Orpinomyces was exclusively found in the rumen of the cows fed the LE diets. This is consistent with the prevalence of this fungal genus previously found in the rumen of cattle fed a high-fiber diet [70]. Even though some of the fungal taxa detected in the present study might be transient, their exclusive interactions with many of the other microbial taxa exclusively for a specific dietary treatment is interesting. It should be noted that even though the numbers of specific nodes of prokaryotes were smaller than those of fungi or protozoa, the total numbers of nodes of prokaryotes were much larger for any of the diets. Thus, ruminal prokaryotes had more extensive interactions than rumen fungi or protozoa. Future research is needed to adequately interpret cooccurrence in the context of lactation performance.

\section{Correlations between microbial genera and lactation traits}

Among the 11 microbial genera that showed a significant and strong correlation with at least one of the lactation traits determined, Ruminobacter, Hanseniaspora, and Isotricha might be positively associated with milk yield, while Coprococcus, Ruminococcus, Mitsuokella, and Hanseniaspora might be associated with milk protein yield. Sphaerochaeta and Saitozyma exhibited negative correlations with two (milk protein content and milk protein yield) and one (milk lactose content) lactation traits, respectively. Little is known about the function of these genera in the rumen. Because correlation does not imply causation, future research is needed to determine their relationship with milk production. Some of these genera, however, may be used as potential biomarkers of lactation traits.

\section{Conclusions}

Dietary energy levels markedly affected the multikingdom microbiota and microbial functions, whereas the energy sources (ground corn vs. steam-flaked corn) had little effect. Some microbial taxa and metabolic pathways were enriched by the high energy level, and they might contribute to the improved lactation performance observed previously. Furthermore, because some of the microbial genera enriched by the high energy diets had exhibited increased predominance in high producing cows, they may be used as biomarkers of one or more of the lactation traits. This study also showed that steam-flaked corn also benefited several microbial taxa, which are associated with high lactation performance. Several uncommon fungi were also affected, especially by the energy level. Specific cooccurrence among different taxa resulted from both the energy level and source (particularly energy level). Future research is warranted to further investigate if the taxonomic drivers identified with FishTaco are the cause of the differential metabolic pathways and how the co-occurrence network can be interpreted in the context of rumen fermentation characteristics and lactation performance.

\section{Supplementary information}

Supplementary information accompanies this paper at https://doi.org/10. 1186/s40104-020-00461-2.

Additional file 1: Figure S1. Relative abundance of the major bacterial and archaeal phyla (A) and genera (B), fungal phyla (C) and genera (D), and protozoal genera (E) (one those each having a relative abundance $\geq 0.1 \%$ in at least one of the dietary treatments are shown). Table S1. Feed and nutrient composition of the diets. Table S2. Primers used for amplicon sequencing.

\section{Abbreviations}

SARA: Subacute rumen acidosis; CS: Corn stover; ME: Metabolizable energy; MP: Microbial protein; SFC: Steam-flaked corn; GC: Ground corn; LE: Low energy; HE: High energy; SRA: Sequence Read Archive; ITS: Internally transcribed spacer; ASV: Amplicon sequence variant; PCoA: Principal coordinates analysis;

SCNIC: Sparse Co-occurrence Network Investigation for Compositional data; CoDiNA: Co-expression Differential Network Analysis; EC: Enzyme Commissions; KEGG: Kyoto Encyclopedia of Genes and Genomes; PCA: Principal components analysis; FishTaco: Functional Shifts' Taxonomic Contributors;

PICRUSt2: Phylogenetic Investigation of Communities by Reconstruction of Unobserved States 2; PERMANOVA: Permutational multivariate analysis of variance; LDA: Linear discriminant analysis; LEfSe: Linear discriminant analysis effect size; NDF: Neutral detergent fiber; FCM: Fat-corrected milk; ECM: Energycorrected milk; VFA: Volatile fatty acid; IMG: Integrated microbial genomes

\section{Authors' contributions}

$Z Y$ and DB conceived the experiment. LM, YM, and XZ conducted the animal experiment. TP analyzed the microbial sequencing data. TP and ZY wrote the manuscript. TP, LM, YM, XZ, DB, and $Z Y$ reviewed the manuscript and read and approved the final manuscript. 


\section{Funding}

This research was partially funded by grants from the National Key Research and Development Program of China (2018YFD0501600), the Scientific Research Project for Major Achievements of The Agricultural Science and Technology Innovation Program (ASTIP) (No. CAAS-ZDXT2019004; CAASXTCX2016011-01; ASTIP-IAS07) and Beijing Dairy Industry Innovation Team (BAIC06-2019), and the USDA National Institute of Food and Agriculture (award number: 2019-67030-29003).

\section{Availability of data and materials}

The raw phylogenetic marker gene sequences were deposited in the NCBI SRA under BioProject PRJNA523854. All the analyzed microbial datasets in the current study are available from the corresponding author on reasonable request.

\section{Ethics approval and consent to participate} Not applicable.

\section{Consent for publication}

All the authors read and agree to the content of this paper and its publication.

\section{Competing interests}

The authors declare no competing interest.

\section{Author details}

'Department of Animal Sciences, The Ohio State University, Columbus, $\mathrm{OH}$, USA. ${ }^{2}$ The State Key Laboratory of Animal Nutrition, Institute of Animal Science, Chinese Academy of Agricultural Sciences, Beijing, P. R. China. ${ }^{3}$ CAAS-ICRAF Joint Lab on Agroforestry and Sustainable Animal Husbandry, Beijing, P. R. China.

\section{Received: 21 January 2020 Accepted: 13 April 2020}

Published online: 22 June 2020

\section{References}

1. Huws SAH, Creevey C, Oyama LB, Mizrahi I, Denman SE, Popova M, et al. Addressing global ruminant agricultural challenges through understanding the rumen microbiome: past, present and future. Front Microbiol. 2018;9:2161.

2. Wanapat M, Foiklang S, Phesatcha K, Paoinn C, Ampapon T, Norrapoke T, et al. On-farm feeding interventions to increase milk production in lactating dairy cows. Tropl Anim Health Prod. 2017;49:829-33.

3. Wallace RJ, Sasson G, Garnsworthy PC, Tapio I, Gregson E, Bani P, et al. A heritable subset of the core rumen microbiome dictates dairy cow productivity and emissions. Sci Adv. 2019;5:eaav8391.

4. Jami E, White BA, Mizrahi I. Potential role of the bovine rumen microbiome in modulating milk composition and feed efficiency. PLoS One. 2014;9: e85423.

5. Shabat SKB, Sasson G, Doron-Faigenboim A, Durman T, Yaacoby S, Miller MEB, et al. Specific microbiome-dependent mechanisms underlie the energy harvest efficiency of ruminants. ISME J. 2016;10:2958-72.

6. Weimer PJ. Redundancy, resilience, and host specificity of the ruminal microbiota: implications for engineering improved ruminal fermentations. Front Microbiol. 2015;6:296.

7. Aguerre MJ, Wattiaux MA, Powell J, Broderick GA, Arndt C. Effect of forageto-concentrate ratio in dairy cow diets on emission of methane, carbon dioxide, and ammonia, lactation performance, and manure excretion. J Dairy Sci. 2011;94:3081-93.

8. Wang B, Mao S, Yang H, Wu Y, Wang J, Li S, et al. Effects of alfalfa and cereal straw as a forage source on nutrient digestibility and lactation performance in lactating dairy cows. J Dairy Sci. 2014;97:7706-15.

9. Zhou X, Zhang Y, Zhao M, Zhang T, Zhu D, Bu D, et al. Effect of dietary energy source and level on nutrient digestibility, rumen microbial protein synthesis, and milk performance in lactating dairy cows. J Dairy Sci. 2015;98: 7209-17.

10. Kolver E, Muller L, Varga G, Cassidy T. Synchronization of ruminal degradation of supplemental carbohydrate with pasture nitrogen in lactating dairy cows. J Dairy Sci. 1998;81:2017-28.

11. Shi H, Li S, Cao Z, Wang Y, Alugongo G, Doane P. Effects of replacing wild rye, corn silage, or corn grain with cao-treated corn Stover and dried distillers grains with solubles in lactating cow diets on performance, digestibility, and profitability. J Dairy Sci. 2015;98:7183-93.

12. Zhu W, Fu Y, Wang B, Wang C, Ye J, Wu Y, et al. Effects of dietary forage sources on rumen microbial protein synthesis and milk performance in early lactating dairy cows. J Dairy Sci. 2013;96:1727-34.

13. Cooke K, Bernard J, West J. Performance of dairy cows fed annual ryegrass silage and corn silage with steam-flaked or ground corn. J Dairy Sci. 2008; 91:2417-22.

14. Theurer C, Huber J, Delgado-Elorduy A, Wanderley R. Invited review: summary of steam-flaking corn or sorghum grain for lactating dairy cows. J Dairy Sci. 1999;82:1950-9.

15. Ferraretto L, Crump P, Shaver R. Effect of cereal grain type and corn grain harvesting and processing methods on intake, digestion, and milk production by dairy cows through a meta-analysis. J Dairy Sci. 2013;96:53350.

16. Yu Z, Morrison M. Improved extraction of pcr-quality community DNA from digesta and fecal samples. BioTechniques. 2004;36:808-12.

17. Bolyen E, Rideout JR, Dillon MR, Bokulich NA, Abnet C, Al-Ghalith GA, et al. Qiime 2: reproducible, interactive, scalable, and extensible microbiome data science. PeerJ Preprints. 2018;6:e27295v2. https://doi.org/10.7287/peerj. preprints.27295v2.

18. Martin M. Cutadapt removes adapter sequences from high-throughput sequencing reads. EMBnet J. 2011;17:10-2.

19. Park T, Mao H, Yu Z. Inhibition of rumen protozoa by specific inhibitors of lysozyme and peptidases in vitro. Front Microbiol. 2019;10:2822.

20. Callahan BJ, McMurdie PJ, Rosen MJ, Han AW, Johnson AJA, Holmes SP. DADA2: high-resolution sample inference from illumina amplicon data. Nat Methods. 2016;13:581-3.

21. Rivers AR, Weber KC, Gardner TG, Liu S, Armstrong SD. ITSxpress: software to rapidly trim internally transcribed spacer sequences with quality scores for marker gene analysis. F1000Research. 2018;7:1418.

22. Bokulich NA, Kaehler BD, Rideout JR, Dillon M, Bolyen E, Knight R, et al. Optimizing taxonomic classification of marker gene amplicon sequences. PeerJ Preprints. 2018;6:90. https://doi.org/10.1186/s40168-018-0470-z.

23. Gysi DM, Fragoso TM, Buskamp V, Almaas E, Nowick K. Comparing multiple networks using the co-expression differential network analysis (CoDiNA). arXiv preprint arXiv. 2018;1802.00828v2.

24. Douglas GM, Maffei VJ, Zaneveld J, Yurgel SN, Brown JR, Taylor CM, et al. PICRUSt2: an improved and extensible approach for metagenome inference. BioRxiv. 2020. https://doi.org/10.1101/672295.

25. Manor O, Borenstein E. Systematic characterization and analysis of the taxonomic drivers of functional shifts in the human microbiome. Cell Host Microbe. 2017;21:254-67.

26. Hammer $\varnothing$, Harper DA, Ryan PD. PAST: paleontological statistics software package for education and data analysis. Palaeontol Electron. 2001;4:9.

27. Segata N, Izard J, Waldron L, Gevers D, Miropolsky L, Garrett WS, et al. Metagenomic biomarker discovery and explanation. Genome Biol. 2011;12:R60.

28. Lee J, Seo J, Lee SY, Ki KS, Seo S. Meta-analysis of factors affecting milk component yields in dairy cattle. J Anim Sci Technol. 2014;56:5

29. Yang W, Beauchemin K. Grain processing, forage-to-concentrate ratio, and forage length effects on ruminal nitrogen degradation and flows of amino acids to the duodenum. J Dairy Sci. 2004;87:2578-90.

30. Dai W, Wang Q, Zhao F, Liu J, Liu H. Understanding the regulatory mechanisms of milk production using integrative transcriptomic and proteomic analyses: improving inefficient utilization of crop by-products as forage in dairy industry. BMC Genomics. 2018;19:403.

31. Kittelmann $\mathrm{S}$, Janssen PH. Characterization of rumen ciliate community composition in domestic sheep, deer, and cattle, feeding on varying diets, by means of pcr-dgge and clone libraries. FEMS Microbiol Ecol. 2011;75:468-81.

32. Belanche A, Doreau M, Edwards JE, Moorby JM, Pinloche E, Newbold CJ. Shifts in the rumen microbiota due to the type of carbohydrate and level of protein ingested by dairy cattle are associated with changes in rumen fermentation. J Nutr. 2012;142:1684-92.

33. Zhang J, Shi H, Wang Y, Li S, Cao Z, Ji S, et al. Effect of dietary forage to concentrate ratios on dynamic profile changes and interactions of ruminal microbiota and metabolites in Holstein heifers. Front Microbiol. 2017:8:2206.

34. Downes J, Munson MA, Radford DR, Spratt DA, Wade WG. Shuttleworthia satelles gen. Nov., sp. Nov., isolated from the human oral cavity. Int I Syst Evol Microbiol. 2002:52:1469-75.

35. Nagaraja T. Microbiology of the rumen. Rumenology: Springer; 2016. p. 39-61. 
36. Xue M, Sun H, Wu X, Liu J. Assessment of rumen microbiota from a large dairy cattle cohort reveals the pan and core bacteriomes contributing to varied phenotypes. Appl Environ Microbiol. 2018;84:e00970-18.

37. Cotta M. Amylolytic activity of selected species of ruminal bacteria. Appl Environ Microbiol. 1988;54:772-6.

38. Klieve A, O'Leary M, McMillen L, Ouwerkerk D. Ruminococcus bromii, identification and isolation as a dominant community member in the rumen of cattle fed a barley diet. J Appl Microbiol. 2007;103:2065-73.

39. Tajima K, Arai S, Ogata K, Nagamine T, Matsui H, Nakamura M, et al. Rumen bacterial community transition during adaptation to high-grain diet. Anaerobe. 2000;6:273-84.

40. Petri RM, Schwaiger T, Penner GB, Beauchemin KA, Forster RJ, McKinnon JJ, et al. Characterization of the core rumen microbiome in cattle during transition from forage to concentrate as well as during and after an acidotic challenge. PLoS One. 2013:8:e83424.

41. Zhang R, Jin W, Feng P, Liu J, Mao S. High-grain diet feeding altered the composition and functions of the rumen bacterial community and caused the damage to the laminar tissues of goats. Animal. 2018;12:2511-20.

42. $\mathrm{Mu} Y$, Lin $X$, Wang $Z$, Hou $Q$, Wang $Y$, Hu Z. High-production dairy cattle exhibit different rumen and fecal bacterial community and rumen metabolite profile than low-production cattle. MicrobiologyOpen. 2019;8:e00673.

43. Xie X, Yang C, Guan LL, Wang J, Xue M, Liu JX. Persistence of cellulolytic bacteria Fibrobacter and Treponema after short-term corn Stover-based dietary intervention reveals the potential to improve rumen fibrolytic function. Front Microbiol. 2018:9:1363.

44. Carlier JP. Moryella. Bergey's manual of systematics of archaea and bacteria; 2015. p. 1-4.

45. Petri RM, Vahmani P, Yang HE, Dugan ME, McAllister TA. Changes in rumen microbial profiles and subcutaneous fat composition when feeding extruded flaxseed mixed with or before hay. Front Microbiol. 2018;9:1055.

46. Lund A. Yeasts and moulds in the bovine rumen. Microbiology. 1974;81: 453-62.

47. Paul SS, Bu D, Xu J, Hyde KD, Yu Z. A phylogenetic census of global diversity of gut anaerobic fungi and a new taxonomic framework. Fungal Divers. 2018:89:253-66

48. Zhang K, Li B, Guo M, Liu G, Yang Y, Wang X, et al. Maturation of the goat rumen microbiota involves three stages of microbial colonization. Animals. 2019:9:1028.

49. Lund A. Yeasts in the rumen contents of musk oxen. Microbiology. 1980; 121:273-6.

50. Fogarty W, Kelly C. Developments in microbial extracellular enzymes. In Wiseman A. Topics in Enzyme and Fermentation Biotechnology. Volume 3. Chichester: Ellis Horwood; 1979. p. 45-102.

51. Cheng Y, Mao S, Liu J, Zhu W. Molecular diversity analysis of rumen methanogenic archaea from goat in eastern China by dgge methods using different primer pairs. Lett Appl Microbiol. 2009;48:585-92.

52. Belanche A, Kingston-Smith AH, Griffith GW, Newbold CJ. A multi-kingdom study reveals the plasticity of the rumen microbiota in response to a shift from non-grazing to grazing diets in sheep. Front Microbiol. 2019;10:122

53. Williams A, Coleman G. The rumen protozoa, Springer series in contemporary bioscience. New York: Springer-Verlag; 1992

54. Hook SE, Steele MA, Northwood KS, Wright A-DG, McBride BW. Impact of high-concentrate feeding and low ruminal $\mathrm{pH}$ on methanogens and protozoa in the rumen of dairy cows. Microb Ecol. 2011;62:94-105.

55. Williams AG. Rumen holotrich ciliate protozoa. Microbiol Rev. 1986;50:25-49.

56. Zhang J, Zhao S, Zhang Y, Sun P, Bu D, Wang J. New primers targeting fulllength ciliate 18s rRNA genes and evaluation of dietary effect on rumen ciliate diversity in dairy cows. Curr Microbiol. 2015;71:650-7.

57. Matsumoto M, Takenaka A, Kobayashi T, Itabashi $\mathrm{H}$. The effects of Epidinium caudatum or Dasytricha ruminantium on the rumen fermentation and nitrogen metabolism in goats. Asian-Australas J Anim Sci. 1989;2:483-4.

58. Söllinger A, Tveit AT, Poulsen M, Noel SJ, Bengtsson M, Bernhardt J, et al. Holistic assessment of rumen microbiome dynamics through quantitative metatranscriptomics reveals multifunctional redundancy during key steps of anaerobic feed degradation. MSystems. 2018;3:e00038-18.

59. Lv X, Chai J, Diao Q, Huang W, Zhuang Y, Zhang N. The signature microbiota drive rumen function shifts in goat kids introduced to solid diet regimes. Microorganisms. 2019;7:516.

60. Li F, Guan LL. Metatranscriptomic profiling reveals linkages between the active rumen microbiome and feed efficiency in beef cattle. Appl Environ Microbiol. 2017;83:e00061-17.
61. Higgins CF. ABC transporters: physiology, structure and mechanism-an overview. Res Microbiol. 2001;152:205-10.

62. Deusch S, Camarinha-Silva A, Conrad J, Beifuss U, Rodehutscord M, Seifert J. A structural and functional elucidation of the rumen microbiome influenced by various diets and microenvironments. Front Microbiol. 2017;8:1605.

63. Kamke J, Kittelmann S, Soni P, Li Y, Tavendale M, Ganesh S, et al. Rumen metagenome and metatranscriptome analyses of low methane yield sheep reveals a sharpea-enriched microbiome characterised by lactic acid formation and utilisation. Microbiome. 2016;4:56.

64. Elolimy AA, Abdelmegeid MK, MCCann JC, Shike DW, Loor JJ. Residual feed intake in beef cattle and its association with carcass traits, ruminal solidfraction bacteria, and epithelium gene expression. J Anim Sci Biotechnol. 2018;9:67.

65. Markowitz VM, Chen I-MA, Palaniappan K, Chu K, Szeto E, Pillay M, et al. IMG 4 version of the integrated microbial genomes comparative analysis system. Nucleic Acids Res. 2013;42:D560-D7.

66. Moriya Y, Itoh M, Okuda S, Yoshizawa AC, Kanehisa M. KAAS: an automatic genome annotation and pathway reconstruction server. Nucleic Acids Res. 2007;35:W182-W5

67. Pitta D, Indugu N, Vecchiarelli B, Rico D, Harvatine KJ. Alterations in ruminal bacterial populations at induction and recovery from diet-induced milk fat depression in dairy cows. J Dairy Sci. 2018:101:295-309.

68. Fujii T, Fang $X$, Inoue H, Murakami K, Sawayama S. Enzymatic hydrolyzing performance of acremonium cellulolyticus and trichoderma reesei against three lignocellulosic materials. Biotechnol Biofuels. 2009;2:24.

69. Coronado-Ruiz C, Avendaño R, Escudero-Leyva E, Conejo-Barboza G, Chaverri $P$, Chavarría M. Two new cellulolytic fungal species isolated from a 19 th-century art collection. Sci Rep. 2018:8:7492.

70. Sirohi SK, Choudhury PK, Puniya AK, Singh D, Dagar SS, Singh N. Ribosomal ITS1 sequence-based diversity analysis of anaerobic rumen fungi in cattle fed on high fiber diet. Ann Microbiol. 2013;63:1571-7.

\section{Ready to submit your research? Choose BMC and benefit from:}

- fast, convenient online submission

- thorough peer review by experienced researchers in your field

- rapid publication on acceptance

- support for research data, including large and complex data types

- gold Open Access which fosters wider collaboration and increased citations

- maximum visibility for your research: over $100 \mathrm{M}$ website views per year

At $\mathrm{BMC}$, research is always in progress.

Learn more biomedcentral.com/submissions 